\title{
ON EXISTENCE OF GLOBAL SOLUTIONS OF SCHRÖDINGER EQUATIONS WITH SUBCRITICAL NONLINEARITY FOR $\widehat{L}^{p}$-INITIAL DATA
}

\author{
RYOSUKE HYAKUNA AND MASAYOSHI TSUTSUMI \\ (Communicated by Matthew J. Gursky)
}

\begin{abstract}
We construct a local theory of the Cauchy problem for the nonlinear Schrödinger equations

$$
\begin{aligned}
& i u_{t}+u_{x x} \pm|u|^{\alpha-1} u=0, \quad x \in \mathbb{R}, \quad t \in \mathbb{R}, \\
& u(0, x)=u_{0}(x)
\end{aligned}
$$

with $\alpha \in(1,5)$ and $u_{0} \in \widehat{L}^{p}(\mathbb{R})$ when $p$ lies in an open neighborhood of 2 . Moreover we prove the global existence for the initial value problem when $p$ is sufficiently close to 2 .
\end{abstract}

\section{INTRODUCTION}

Consider the following Cauchy problem:

$$
\left(\mathbf{N L S}^{\alpha}\right)\left\{\begin{array}{l}
i u_{t}+u_{x x} \pm|u|^{\alpha-1} u=0, \quad t \in \mathbb{R}, \\
u(0, x)=u_{0}(x), \quad x \in \mathbb{R},
\end{array}\right.
$$

where $u: \mathbb{R}_{t} \times \mathbb{R}_{x} \rightarrow \mathbb{C}$ is an unknown function and $1<\alpha<5$. We are interested in the problem of whether or not a global solution of $\left(\mathbf{N L S}^{\alpha}\right)$ exists when $u_{0} \in L^{p}(\mathbb{R})$ or $u_{0} \in \widehat{L}^{p}(\mathbb{R})$, where $\widehat{L}^{p}$ is defined by

$$
\widehat{L}^{p}:=\left\{\varphi: \widehat{\varphi} \in L^{p^{\prime}}\right\}
$$

and we note that by the Hausdorff-Young inequality, $L^{p} \subseteq \widehat{L}^{p}$ if $p \leq 2$ and $\widehat{L}^{p} \subseteq L^{p}$ if $p \geq 2$. As is well-known, the Cauchy problem $\left(\mathbf{N L S}^{\alpha}\right)$ is globally well-posed if $p=2$. When $p \neq 2$, although not many authors study this subject, there are some recent works in terms of the $L^{p}$ - and $\widehat{L}^{p}$-local well-posedness of $\left(\mathbf{N L S}^{\alpha}\right)$ in the cubic case $(\alpha=3)$ : Zhou 13 obtained local well-posedness in $L^{p}$ for $1<p<2$. Grünrock [5] proved the local well-posedness in $\widehat{L}^{p}$ for $1<p<\infty$ and global existence for $\frac{5}{3}<p<2$. For general $\alpha \in(1,5)$, the authors studied the case of $u_{0} \in L^{p}$ with $p<2$ and constructed a global solution of $\left(\mathbf{N L S}^{\alpha}\right)$ in $[6$ ] when $1<\alpha \leq 3$ and $p$ is close to 2 .

In this paper we investigate $\left(\mathbf{N L S}^{\alpha}\right)$ when $u_{0} \in \widehat{L}^{p}$ and $p$ is near 2 . Two types of estimates play an important role in our study. One is the inequality of type

$$
\left\|e^{i t \partial_{x}^{2}} f\right\|_{L^{q} L^{r}} \lesssim\|f\|_{\widehat{L}^{p}}, \quad \frac{2}{q}+\frac{1}{r}=\frac{1}{p} .
$$

Received by the editors December 14, 2010 and, in revised form, May 10, 2011. 2010 Mathematics Subject Classification. Primary 35Q55, 35Q41. 
When $q=r$ and $p>\frac{4}{3}$, the inequality (2) is valid and is known as the FeffermanStein estimate, which goes back to 3 . It is not difficult to see that the inequality leads to the local well-posedness of the cubic NLS when $\frac{4}{3}<p<2$ (see [4]). In the present paper we prove (2) for more general pairs, especially for the case of $q \neq r$ in order to adapt to the setting of our paper. Moreover, we also use the Strichartz estimates for inhomogeneous equations:

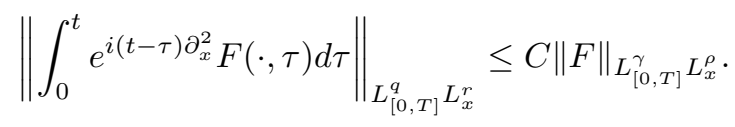

Although this estimate is well-known when both $(q, r)$ and $\left(\gamma^{\prime}, \rho^{\prime}\right)$ are admissible, we need a more general version. Indeed, when $p>2$, we seek a solution of $\left(\mathbf{N L S}^{\alpha}\right)$ in $L_{[0, T]}^{q}\left(L^{r}\right)$ spaces with $q>\frac{2 r}{r-2}$ and in such cases the admissible version of (3) is no longer applicable. Here in this paper, we will use the result by Kato [7]. For updated results of the Strichartz estimate of type (3) including multidimensional and endpoint cases, see Vilela [11.

Once we construct a local theory of $\left(\mathbf{N L S}^{\alpha}\right)$, we then extend these solutions globally, especially when $p$ is close enough to 2 . Our strategy is motivated by the work of Vargas and Vega 12, and here we split the initial data $u_{0} \in \widehat{L}^{p}$ using an interpolation lemma for $\widehat{L}^{p}$-spaces:

$$
u_{0}=\varphi_{N}+\psi_{N} \quad \text { and } \quad\left\|\varphi_{N}\right\|_{L^{2}} \sim N^{\frac{1-\theta}{\theta}}, \quad\left\|\psi_{N}\right\|_{\widehat{L}^{p_{0}}} \lesssim N^{-1}, \quad N>1
$$

with

$$
\frac{1}{p^{\prime}}=\frac{1}{p_{\theta}^{\prime}}=\frac{1-\theta}{p_{0}^{\prime}}+\frac{\theta}{2}, \quad 0<\theta<1,
$$

where $p_{0}$ will be determined later. Then we apply Bourgain's method [1] to find $\theta$ for which a global solution of $\left(\mathbf{N L S}^{\alpha}\right)$ with $u_{0} \in \widehat{L}^{p_{\theta}}$ exists.

To state our results we define for $\alpha \in(1,5)$,

$$
r_{\alpha}:=\left\{\begin{array}{lll}
\alpha+1 & \text { if } & \alpha \in(1,3) \\
4 & \text { if } & \alpha \in\left[3, \frac{11}{3}\right] \\
\frac{12}{11} \alpha & \text { if } & \alpha \in\left(\frac{11}{3}, 5\right)
\end{array}\right.
$$

and for $p>1, q_{\alpha, p}$ is defined by

$$
\frac{2}{q_{\alpha, p}}+\frac{1}{r_{\alpha}}=\frac{1}{p}
$$

Our main result is as follows.

Theorem 1. (i) Let $u_{0} \in \widehat{L}^{p}(\mathbb{R})$ with

$$
2>p>\max \left(\frac{4}{3}, \frac{\alpha-1}{2}, \frac{\alpha+1}{\alpha}\right) .
$$

Then there exist $T>0$ and a solution $u$ of the Cauchy problem $\left(\mathbf{N L} \mathbf{S}^{\alpha}\right)$ in $[-T, T]$ with

$$
u \in C\left([-T, T]: \widehat{L}^{p}(\mathbb{R})\right) \cap L^{q_{\alpha, p}}\left([-T, T]: L^{r_{\alpha}}(\mathbb{R})\right) .
$$

Moreover, the mapping $u_{0} \mapsto u(t)$ is locally Lipschitz from $\widehat{L}^{p}$ to $C\left([-T, T]: \widehat{L}^{p}\right) \cap$ $L^{q_{\alpha, p}}\left([-T, T]: L^{r_{\alpha}}\right)$. 
(ii) Let $u_{0} \in \widehat{L}^{p}$ with

$$
2<p<\min \left(\frac{2(\alpha+1)}{\alpha-1}, \alpha+1\right) .
$$

Then there exist $T>0$ and a solution $u$ of the Cauchy problem $\left(\mathbf{N L S}^{\alpha}\right)$ in $[-T, T]$ with

$$
u \in C\left([-T, T]: \widehat{L}^{p}(\mathbb{R})\right) \cap L^{q}\left([-T, T]: L^{\alpha+1}(\mathbb{R})\right)
$$

where

$$
\frac{2}{q}+\frac{1}{\alpha+1}=\frac{1}{p}
$$

Moreover, the mapping $u \mapsto u(t)$ is locally Lipschitz from $\widehat{L}^{p}$ to $C\left([-T, T]: \widehat{L}^{p}\right) \cap$ $L^{q}\left([-T, T]: L^{\alpha+1}\right)$.

Furthermore, the solution in Theorem 1 can be extended globally when $p$ is near 2. For $\alpha \in(1,5)$ we define

$$
q_{\alpha}^{\min }:=\left\{\begin{array}{lll}
\frac{2(\alpha+1)}{\alpha-1} & \text { if } & \alpha \in(1,3), \\
4 & \text { if } & \alpha \in\left[3, \frac{10}{3}\right], \\
\frac{8 \alpha}{10-\alpha} & \text { if } & \alpha \in\left(\frac{10}{3}, \frac{11}{3}\right], \\
\frac{24}{19} \alpha & \text { if } & \alpha \in\left(\frac{11}{3}, 5\right) .
\end{array}\right.
$$

Our global result is the following.

Theorem 2. (i) Let $u_{0} \in \widehat{L}^{p}(\mathbb{R})$ with

$$
2>p> \begin{cases}\frac{\alpha+1}{\alpha} & \text { if } 1<\alpha<\frac{3+\sqrt{57}}{6}, \\ \frac{5 \alpha+3}{2(\alpha+2)} & \text { if } \frac{3+\sqrt{57}}{6} \leq \alpha<3, \\ \frac{9(\alpha-1)}{2(2 \alpha-1)} & \text { if } 3 \leq \alpha \leq \frac{10}{3} \\ \frac{(\alpha-1)(3 \alpha+5)}{2\left(\alpha^{2}-2 \alpha+5\right)} & \text { if } \frac{10}{3} \leq \alpha<5 .\end{cases}
$$

Then there exists $\tilde{q} \in\left(q_{\alpha}^{\mathrm{min}}, q_{\alpha, p}\right)$ such that the Cauchy problem $\left(\mathbf{N L S}^{\alpha}\right)$ has a unique global solution $u$ of the form

$$
u=v+w \in L_{\text {loc }}^{q_{\alpha, 2}}\left(\mathbb{R}: L^{r_{\alpha}}(\mathbb{R})\right) \cap L_{\text {loc }}^{\infty}\left(\mathbb{R}: L^{2}(\mathbb{R})\right)+L_{\text {loc }}^{\tilde{q}}\left(\mathbb{R}: L^{r_{\alpha}}(\mathbb{R})\right) .
$$

(ii) Let $u_{0} \in \widehat{L}^{p}(\mathbb{R})$ with

$$
2<p< \begin{cases}\alpha+1 \quad \text { if } \quad 1<\alpha \leq \frac{1+\sqrt{41}}{4} \\ \frac{3 \alpha+5}{2 \alpha} \quad \text { if } \quad \frac{1+\sqrt{41}}{4}<\alpha<5\end{cases}
$$

Then there is a sufficiently large number $Q$ such that the Cauchy problem $\left(\mathbf{N L S}^{\alpha}\right)$ has a unique global solution $u$ of the form

$$
u=v+w \in L_{\text {loc }}^{\frac{4(\alpha+1)}{\alpha-1}}\left(\mathbb{R}: L^{\alpha+1}(\mathbb{R})\right) \cap L_{\text {loc }}^{\infty}\left(\mathbb{R}: L^{2}(\mathbb{R})\right)+L_{\text {loc }}^{Q}\left(\mathbb{R}: L^{\alpha+1}(\mathbb{R})\right) .
$$

We give some notation which is used throughout this paper.

Notation. (i) For an arbitrary $a \in[1, \infty], a^{\prime}$ is the conjugate of $a$, namely

$$
\frac{1}{a}+\frac{1}{a^{\prime}}=1
$$

(ii) Let $I \subseteq \mathbb{R}$ be an interval. For the sake of simplicity, we abbreviate $L^{q}(I$ : $\left.L^{r}(\mathbb{R})\right)$ to $L_{I}^{q} L_{x}^{r}$. In particular, we write $L_{\mathbb{R}}^{q} L^{r}=L^{q} L^{r}$, when $I=\mathbb{R}$. 
(iii) Let $f, F$ be functions on $\mathbb{R}_{x}$ and $\mathbb{R}_{t} \times \mathbb{R}_{x}$, respectively. Denote $\hat{f}, \hat{F}$ by the Fourier transform of $f, F$ with respect to the space variable.

(iv) Define the two complex-valued functions $G$ and $\tilde{G}$ by

$$
\begin{aligned}
& G(v, w)=|v+w|^{\alpha-1}(v+w)-|v|^{\alpha-1} v, \quad v, w \in \mathbb{C}, \\
& \tilde{G}\left(v, w_{1}, w_{2}\right)=G\left(v, w_{1}\right)-G\left(v, w_{2}\right), \quad v, w_{1}, w_{2} \in \mathbb{C} .
\end{aligned}
$$

Note that $G(v, w)=\tilde{G}(v, w, 0)$.

(v) For any $T>0$, put $I_{T}=[0, T]$.

\section{Preliminaries}

In this section we mainly treat Strichartz type estimates for homogeneous and inhomogeneous equations and their corollaries. We introduce the geometric notation of Kato [7, 8]. Consider the closed unit square $R$ in $\mathbb{R}^{2}$ :

$$
R:=\left\{(x, y) \in \mathbb{R}^{2}: 0 \leq x, y \leq 1\right\} .
$$

Then, we introduce some special points in $R$ :

$$
\begin{gathered}
O=(0,0), B=\left(\frac{1}{2}, 0\right), C=\left(0, \frac{1}{4}\right), E=\left(0, \frac{1}{2}\right), F=\left(\frac{1}{4}, \frac{1}{4}\right), \\
O^{\prime}=(1,1), B^{\prime}=\left(\frac{1}{2}, 1\right), C^{\prime}=\left(1, \frac{3}{4}\right), E^{\prime}=\left(1, \frac{1}{2}\right), F^{\prime}=\left(\frac{3}{4}, \frac{3}{4}\right),
\end{gathered}
$$

and define the triangles $\widehat{T}_{1}:=\triangle O B E$ and $\widehat{T}_{2}:=\triangle O^{\prime} B^{\prime} E^{\prime}$, which are open except that $B$ and $B^{\prime}$ are included. Also we define $\widehat{T}_{3}$ as the triangle $B F C$ which is open except that the segment $\left\{\left(x, \frac{1}{4}\right): 0<x<\frac{1}{4}\right\}$ is included. Obviously, we have

$$
\begin{aligned}
B C & :=\left\{(x, y) \in R:\left(\frac{1}{y}, \frac{1}{x}\right) \text { is admissible }\right\}, \\
B^{\prime} C^{\prime} & :=\left\{(x, y) \in R:\left(\frac{1}{y^{\prime}}, \frac{1}{x^{\prime}}\right) \text { is admissible }\right\} .
\end{aligned}
$$

Interpolating the operator $e^{i t \partial_{x}^{2}} \circ \mathcal{F}^{-1}$ between $O$ and any point on the admissible line $B C$, we get

Lemma 3. Let $p \geq 2$. Let $\left(\frac{1}{r}, \frac{1}{q}\right)$ lie in the closed triangle $\triangle O B C$ and satisfy

$$
\frac{2}{q}+\frac{1}{r}=\frac{1}{p}
$$

Then, there is a positive constant $C_{1}>0$ such that we have

$$
\left\|e^{i t \partial_{x}^{2}} f\right\|_{L_{I_{T}}^{q} L^{r}} \leq C_{1}\|f\|_{\widehat{L}^{p}}
$$

for any $T>0$ and $f \in \widehat{L}^{p}$.

As we noted in the introduction, the inequality (7) still holds true when $q=r$ and $\frac{4}{3}<p<2$. We then generalize this inequality for pairs with $\left(\frac{1}{r}, \frac{1}{q}\right) \in \widehat{T}_{3}$. This off-diagonal generalization is one of our key inequalities to both our local and global results. We first need the following lemma.

Lemma 4. Suppose $4<r<\infty$ and

$$
\frac{1}{2}+\frac{1}{r}=\frac{1}{p}
$$


Then, there is a positive constant $C_{1}>0$ such that we have

$$
\left\|e^{i t \partial_{x}^{2}} f\right\|_{L_{I_{T}}^{4} L^{r}} \leq C_{1}\|f\|_{\widehat{L}^{p}}
$$

for any $T>0$ and $f \in \widehat{L}^{p}$.

Proof. We follow the proofs of Lemmas 3.1 and 3.3 in [4, where the Airy version of (8) is shown. By the Sobolev embedding theorem and the Plancherel identity, we have

$$
\begin{aligned}
\left\|e^{i t \partial_{x}^{2}} f\right\|_{L_{t}^{4} L_{x}^{r}}^{4}=\left\|\left|e^{i t \partial_{x}^{2}} f\right|^{2}\right\|_{L_{t}^{2} L_{x}^{\frac{r}{2}}}^{2} & \leq C\left\|I^{\varepsilon_{r}}\left|e^{i t \partial_{x}^{2}} f\right|\right\|_{L_{t}^{2} L_{x}^{2}}^{2} \\
& =\left\||\xi|^{\varepsilon_{r}} \cdot\left(\widehat{\left(e^{i t \partial_{x}^{2}} f\right.}\right) *\left(\overline{\left(\bar{e}^{i t \partial_{x}^{2}} f\right.}\right)\right\|_{L_{t}^{2} L_{\xi}^{2}}^{2},
\end{aligned}
$$

where $I^{\varepsilon_{r}} \varphi$ is defined by $\widehat{\left(I^{\varepsilon_{r}} \varphi\right)}(\xi):=|\xi|^{\varepsilon_{r}} \widehat{\varphi}(\xi)$ and $\varepsilon_{r}:=\frac{1}{2}-\frac{2}{r}$. The right-hand side of the above inequality equals

$$
\begin{aligned}
& \iint d t d \xi|\xi|^{2 \varepsilon_{r}}\left|\int e^{i t(\xi-\eta)^{2}} \widehat{f}(\xi-\eta) e^{-i t \eta^{2}} \overline{\widehat{f}(-\eta)} d \eta\right|^{2} \\
& =\iint d t d \xi|\xi|^{2 \varepsilon_{r}}\left(\int e^{i t\left(\xi-\eta_{1}\right)^{2}} \widehat{f}\left(\xi-\eta_{1}\right) e^{-i t \eta_{1}^{2}} \overline{\widehat{f}\left(-\eta_{1}\right)} d \eta_{1}\right) \\
& \times \overline{\left(\int e^{i t\left(\xi-\eta_{2}\right)^{2}} \widehat{f}\left(\xi-\eta_{2}\right) e^{-i t \eta_{2}^{2}} \overline{\widehat{f}\left(-\eta_{2}\right)} d \eta_{2}\right)} \\
& =\iint d t d \xi|\xi|^{2 \varepsilon_{r}} \int e^{2 i t \xi\left(\eta_{2}-\eta_{1}\right)} \widehat{f}\left(\xi-\eta_{1}\right) \overline{\widehat{f}\left(-\eta_{1}\right)} \widehat{\hat{f}\left(\xi-\eta_{2}\right)} \widehat{f}\left(-\eta_{2}\right) d \eta_{1} d \eta_{2} \\
& =\int d \xi|\xi|^{2 \varepsilon_{r}} \int \delta\left(2 \xi\left(\eta_{1}-\eta_{2}\right)\right) \widehat{f}\left(\xi-\eta_{1}\right) \overline{\widehat{f}\left(-\eta_{1}\right)} \overline{\widehat{f}\left(\xi-\eta_{2}\right)} \widehat{f}\left(-\eta_{2}\right) d \eta_{1} d \eta_{2} \\
& =\int d \xi d \eta_{1}|\xi|^{2 \varepsilon_{r}-1}\left|\widehat{f}\left(\xi-\eta_{1}\right)\right|^{2}\left|\widehat{f}\left(-\eta_{1}\right)\right|^{2},
\end{aligned}
$$

which can be controlled by

$$
\left\||\widehat{f}|^{2}\right\|_{L^{\frac{p^{\prime}}{2}}} \cdot\left\||\widehat{f}|^{2} *|\cdot|^{2 \varepsilon_{r}-1}\right\|_{L^{\frac{p^{\prime}}{p^{\prime}-2}}} \leq\|\widehat{f}\|_{L^{p^{\prime}}}^{4}
$$

by Hölder's inequality and the Hardy-Littlewood-Sobolev theorem. This completes the proof.

Using interpolation between Lemma 4 and the linear Strichartz estimate for $L^{2}$-data, we obtain the following inequality.

Theorem 5. Suppose that $\left(\frac{1}{r}, \frac{1}{q}\right) \in \widehat{T}_{3}$. Then there is a positive constant $C_{1}>0$ such that we have

$$
\left\|e^{i t \partial_{x}^{2}} f\right\|_{L_{I_{T}}^{q} L^{r}} \leq C_{1}\|f\|_{\widehat{L}^{p}}
$$

for any $T>0$ and $f \in \widehat{L}^{p}$.

Another key estimate is the inhomogeneous Strichartz estimate for Hölder exponents which are not necessarily admissible. 
Theorem 6. Suppose that $\left(\frac{1}{r}, \frac{1}{q}\right) \in \widehat{T}_{1},\left(\frac{1}{\rho}, \frac{1}{\gamma}\right) \in \widehat{T}_{2}$ and

$$
\frac{2}{\gamma}+\frac{1}{\rho}=2+\frac{2}{q}+\frac{1}{r} \text {. }
$$

Then, there is a constant $C_{2}>0$ such that the estimate

$$
\left\|\int_{0}^{t} e^{i(t-\tau) \partial_{x}^{2}} F(\cdot, \tau) d \tau\right\|_{L_{I_{T}}^{q} L_{x}^{r}} \leq C_{2}\|F\|_{L_{I_{T}}^{\gamma} L_{x}^{\rho}}
$$

is valid for any $T>0$ and $F \in L_{I_{T}}^{\gamma} L_{x}^{\rho}$.

Proof. See [7, Theorem 2.1].

Proposition 7. We get the following inequalities:

(i) Let $q>\max \left(\frac{2(\alpha+1)}{\alpha-1}, \frac{2(\alpha+1)(\alpha-1)}{\alpha+3}, \alpha-1\right)$. Then, there exists $C_{3}>0$ such that for any $T>0$ we have

$$
\begin{aligned}
& \left\|\int_{0}^{t} e^{i(t-\tau) \partial_{x}^{2}} \tilde{G}\left(v, w_{1}, w_{2}\right)(\cdot, \tau) d \tau\right\|_{L_{I_{T}}^{q} L^{\alpha+1}} \\
& \quad \leq C_{3}\left[T^{\frac{5-\alpha}{4}}\|v\|_{L_{I_{T}}^{\frac{4-1(\alpha+1)}{\alpha-1}}}^{L^{\alpha+1}}\left\|w_{1}-w_{2}\right\|_{L_{I_{T}}^{q} L^{\alpha+1}}\right. \\
& \left.\quad+T^{1-\frac{\alpha-1}{2(\alpha+1)}-\frac{\alpha-1}{q}}\left\{\left\|w_{1}\right\|_{L_{I_{T}}^{q} L^{\alpha+1}}^{\alpha-1}+\left\|w_{2}\right\|_{L_{I_{T}}^{q} L^{\alpha+1}}^{\alpha-1}\right\} \times\left\|w_{1}-w_{2}\right\|_{L_{I_{T}}^{q} L^{\alpha+1}}\right]
\end{aligned}
$$

for all $v \in L_{I_{T}}^{\frac{4(\alpha+1)}{\alpha-1}} L^{\alpha+1}, w_{1}, w_{2} \in L_{I_{T}}^{q} L^{\alpha+1}$.

(ii) Let $3 \leq \alpha \leq \frac{11}{3}$ and $q>\max \left\{\frac{8}{\alpha-1}, \frac{8(\alpha-1)}{9-\alpha}\right\}$. Then, there exists $C_{3}>0$ such that for any $T>0$ we have

$$
\left\|\int_{0}^{t} e^{i(t-\tau) \partial_{x}^{2}} \tilde{G}\left(v, w_{1}, w_{2}\right)(\cdot, \tau) d \tau\right\|_{L_{I_{T} L^{4}}^{q}} \leq C_{3}\left[T^{\frac{5-\alpha}{4}}\|v\|_{L_{I_{T}}^{8} L^{4}}^{\alpha-1}\left\|w_{1}-w_{2}\right\|_{L_{I_{T}}^{q} L^{4}}\right.
$$

$$
\left.+T^{\frac{9-\alpha}{8}-\frac{\alpha-1}{q}}\left\{\left\|w_{1}\right\|_{L_{I_{T}}^{q} L^{4}}^{\alpha-1}+\left\|w_{2}\right\|_{L_{I_{T}}^{q} L^{4}}^{\alpha-1}\right\} \times\left\|w_{1}-w_{2}\right\|_{L_{I_{T}}^{q} L^{4}}\right]
$$

for all $v \in L_{I_{T}}^{8} L^{4}, w_{1}, w_{2} \in L_{I_{T}}^{q} L^{4}$.

(iii) Let $\alpha>\frac{11}{3}$ and $q>\max \left(\frac{24 \alpha}{11(\alpha-1)}, \frac{24 \alpha(\alpha-1)}{13 \alpha+11}\right)$. Then, there exists $C_{3}>0$ such that for any $T>0$ we have

$$
\begin{aligned}
& \left\|\int_{0}^{t} e^{i(t-\tau) \partial_{x}^{2}} \tilde{G}\left(v, w_{1}, w_{2}\right)(\cdot, \tau) d \tau\right\|_{L_{I_{T}}^{q} L^{\frac{12}{11} \alpha}} \\
& \quad \leq C_{3}\left[T^{\frac{5-\alpha}{4}}\|v\|_{L_{I_{T}}^{\alpha-1} \frac{24 \alpha}{6 \alpha-11} L^{\frac{12}{11} \alpha}}^{\alpha}\left\|w_{1}-w_{2}\right\|_{L_{I_{T}}^{q} L^{\frac{12}{11} \alpha}}\right.
\end{aligned}
$$

$$
\left.+T^{\frac{13 \alpha+11}{24 \alpha}-\frac{\alpha-1}{q}}\left\{\left\|w_{1}\right\|_{L_{I_{T}}^{q} L_{12}^{11} \alpha}^{\alpha-1}+\left\|w_{2}\right\|_{L_{I_{T}}^{q} L^{\frac{12}{11} \alpha}}^{\alpha-1}\right\} \times\left\|w_{1}-w_{2}\right\|_{L_{I_{T}}^{q} L^{\frac{12}{11} \alpha}}\right]
$$


for all $v \in L_{I_{T}}^{\frac{24 \alpha}{6 \alpha-11}} L^{\frac{12}{11} \alpha}, w_{1}, w_{2} \in L_{I_{T}}^{q} L^{\frac{12}{11} \alpha}$.

Proof. All the inequalities are proven in the same way. We first use Theorem 6 to obtain

$$
\left\|\int_{0}^{t} e^{i(t-\tau) \partial_{x}^{2}} \tilde{G}\left(v, w_{1}, w_{2}\right)(\cdot, \tau) d \tau\right\|_{L_{I_{T}}^{q} L^{\alpha+1}} \leq C\left\|\tilde{G}\left(v, w_{1}, w_{2}\right)\right\|_{L_{I_{T}}^{A} L^{\frac{\alpha+1}{\alpha}}}
$$

for $1<\alpha<5$ and

$$
\left\|\int_{0}^{t} e^{i(t-\tau) \partial_{x}^{2}} \tilde{G}\left(v, w_{1}, w_{2}\right)(\cdot, \tau) d \tau\right\|_{L_{I_{T}}^{q} L^{r_{\alpha}}} \leq C\left\|\tilde{G}\left(v, w_{1}, w_{2}\right)\right\|_{L_{I_{T}}^{B} L^{\frac{r_{\alpha}}{\alpha}}}
$$

for $\alpha \in\left[\frac{11}{3}, 5\right)$, where

$$
A=\left(1+\frac{1}{q}-\frac{\alpha-1}{2(\alpha+1)}\right)^{-1}, \quad B=\left(1+\frac{1}{q}-\frac{\alpha-1}{2 r_{\alpha}}\right)^{-1} .
$$

Then, by the inequality

$$
\left|\tilde{G}\left(v, w_{1}, w_{2}\right)\right| \lesssim\left(|v|^{\alpha-1}+\left|w_{1}\right|^{\alpha-1}+\left|w_{2}\right|^{\alpha-1}\right)\left|w_{1}-w_{2}\right|
$$

and Hölder's inequality, we get the inequalities (12)-(14). One can easily check that the above procedure is justified by carefully checking that the indices in the calculation are in an appropriate range under the assumptions of the proposition.

To obtain global results we need the following.

Proposition 8. (i) Let $q>\frac{4 \alpha(\alpha+1)}{3 \alpha+5}$. Then, there exists $C_{3}>0$ such that for any $T>0$ we have

$$
\begin{aligned}
& \left\|\int_{0}^{t} e^{i(t-\tau) \partial_{x}^{2}} \tilde{G}(v, w, 0)(\cdot, \tau) d \tau\right\|_{L_{I_{T}}^{\infty} L^{2}} \\
& \leq C_{3}\left[T^{\frac{5-\alpha}{4}+\frac{\alpha-1}{4(\alpha+1)}-\frac{1}{q}}\|v\|_{L_{I_{T}}^{\alpha-1}}^{\frac{4(\alpha+1)}{\alpha-1}} L_{L^{\alpha+1}}\|w\|_{\left.L_{I_{T}}^{q} L^{\alpha+1}+T^{\frac{3 \alpha+5}{4(\alpha+1)}-\frac{\alpha}{q}}\|w\|_{L_{I_{T}}^{q} L^{\alpha+1}}^{\alpha}\right]}\right]
\end{aligned}
$$

for all $v \in L_{I_{T}}^{\frac{4(\alpha+1)}{\alpha-1}} L^{\alpha+1}$ and $w \in L_{I_{T}}^{q} L^{\alpha+1}$.

(ii) Let $3 \leq \alpha<\frac{11}{3}$ and $q>\frac{8 \alpha}{10-\alpha}$. Then, there exists $C_{3}>0$ such that for any $T>0$ we have

$$
\begin{aligned}
\left\|\int_{0}^{t} e^{i(t-\tau) \partial_{x}^{2}} \tilde{G}(v, w, 0)(\cdot, \tau) d \tau\right\|_{L_{I_{T}}^{\infty} L^{2}} \leq C_{3}\left[T^{\frac{11-2 \alpha}{8}-\frac{1}{q}}\|v\|_{L_{I_{T}}^{8} L^{4}}^{\alpha-1}\|w\|_{L_{I_{T}}^{q} L^{4}}\right. \\
\left.+T^{\frac{10-\alpha}{8}-\frac{\alpha}{q}}\|w\|_{L_{I_{T}}^{q} L^{4}}^{\alpha}\right]
\end{aligned}
$$

for all $v \in L_{I_{T}}^{8} L^{4}$ and $w \in L_{I_{T}}^{q} L^{4}$. 
(iii) Let $\alpha>\frac{11}{3}$ and $q>\frac{24 \alpha}{19}$. Then, there exists $C_{3}>0$ such that for any $T>0$ we have

$$
\begin{aligned}
& \left\|\int_{0}^{t} e^{i(t-\tau) \partial_{x}^{2}} \tilde{G}(v, w, 0)(\cdot, \tau) d \tau\right\|_{L_{I_{T}}^{\infty} L^{2}} \\
& \quad \leq C_{3}\left[T^{\frac{-6 \alpha^{2}+36 \alpha-11}{24 \alpha}-\frac{1}{q}\|v\|_{L_{I_{T}}^{\sigma-1} \frac{24 \alpha}{6 \alpha-11} L^{\frac{12}{11} \alpha}}^{\alpha-1}}\|w\|_{L_{I_{T}}^{q} L^{\frac{12}{11} \alpha}}+T^{\frac{19}{24}-\frac{\alpha}{q}}\|w\|_{L_{I_{T}}^{q} L^{\frac{12}{11} \alpha}}^{\alpha}\right]
\end{aligned}
$$

for all $v \in L_{I_{T}}^{\frac{24 \alpha}{6 \alpha-11}} L^{\frac{12}{11} \alpha}$ and $w \in L_{I_{T}}^{q} L^{\frac{12}{11} \alpha}$.

Proposition 9. Suppose that $v: \mathbb{R} \times \mathbb{R} \rightarrow \mathbb{C}$ with $\varphi(\cdot):=v(0, \cdot) \in L_{x}^{2}$ solves

$$
i v_{t}+v_{x x} \pm|v|^{\alpha-1} v=0
$$

and that $(q, r)$ is an admissible pair. Then, there are positive constants $K_{1}, K_{2}$ depending only on $\alpha$ such that

$$
\|v\|_{L_{I_{\delta}}^{q} L^{r}} \leq K_{1}\|\varphi\|_{L_{x}^{2}}
$$

for any $\delta \in\left[0,\left(K_{2}\|\varphi\|_{L_{x}^{2}}\right)^{-\frac{4(\alpha-1)}{5-\alpha}}\right]$, provided that

(i) $1<\alpha<5$ and $q=\frac{4(\alpha+1)}{\alpha-1}, r=\alpha+1$,

(ii) $3<\alpha<5$ and $q=q_{\alpha, 2}, r=r_{\alpha}$.

Proof. See [6], Lemma 2.4 .

We close the section with a useful result from interpolation theory.

Lemma 10. Suppose that $0<q_{0}<q_{1} \leq \infty, 0<\theta<1$ and $q>0$ is defined by

$$
\frac{1}{q}=\frac{1-\theta}{q_{0}}+\frac{\theta}{q_{1}}
$$

Then,

$$
L^{q} \subset L^{q_{0}}+L^{q_{1}} .
$$

Furthermore, for any $f \in L^{q}$ and for any $t>0$ one can find $f_{0}^{t} \in L^{q_{0}}$ and $f_{1}^{t} \in L^{q_{1}}$ with $f=f_{0}^{t}+f_{1}^{t}$ satisfying

$$
c t^{-\theta} \max \left\{\left\|f_{0}^{t}\right\|_{L^{q_{0}}}, t\left\|f_{1}^{t}\right\|_{L^{q_{1}}}\right\} \leq\|f\|_{L^{q}}
$$

for some positive constant $c$ depending only on $q_{0}, q_{1}$.

For a proof, see $[9], 6]$.

\section{Proof of Theorem 1}

Consider the integral equation

$$
u(t)=e^{i t \partial_{x}^{2}} u_{0} \pm i \int_{0}^{t} e^{i(t-\tau) \partial_{x}^{2}}\left(|u|^{\alpha-1} u\right) d \tau
$$

and put

$$
\mathcal{V}^{q, r}:=C\left([0, T]: \widehat{L}^{p}\right) \cap L_{[0, T]}^{q} L^{r}
$$

with $\frac{2}{q}+\frac{1}{r}=\frac{1}{p}$. It is enough to show that the $\mathcal{V}^{q, r}$-norm of the right-hand side of (21) can be controlled by

$$
\left\|u_{0}\right\|_{\widehat{L}^{p}}+T^{\beta}\|u\|_{L_{[0, T]}^{q}}^{\alpha} L^{r}
$$


for some $\beta>0$ and $(q, r)$ (see Proposition 1.1 and a sketch of its proof in [4]).

We first prove $(i)$. Let $2>p>\max \left(\frac{4}{3}, \frac{\alpha-1}{2}, \frac{\alpha+1}{\alpha}\right)$ and $q=q_{\alpha, p}, r=r_{\alpha}$. Then the $\mathcal{V}^{q, r}$-norm of the linear part is bounded from above by $\left\|u_{0}\right\|_{\widehat{L}^{p}}$ by the trivial equality $\left\|e^{i t \partial_{x}^{2}} u_{0}\right\|_{\widehat{L}^{p}}=\left\|u_{0}\right\|_{\widehat{L}^{p}}$ and the generalized Fefferman-Stein inequality since $\left(\frac{1}{r_{\alpha}}, \frac{1}{q_{\alpha, p}}\right) \in \widehat{T}_{3}$. For the nonlinear part, it is not difficult to check that $q_{\alpha, p}$ satisfies the assumption of Proposition 7 . Thus we have

$$
\left\|\int_{0}^{t} e^{i(t-\tau) \partial_{x}^{2}}\left(|u|^{\alpha-1} u\right) d \tau\right\|_{L_{[0, T]}^{q_{\alpha, p} L^{r_{\alpha}}}} \leq C T^{\beta}\|u\|_{L_{[0, T]}^{q_{\alpha, p} L^{r_{\alpha}}}}^{\alpha},
$$

where $\beta$ is the exponent of $T$ in the second term on the right-hand side of (12)-(14). Moreover, by the duality of Lemma 3, we have

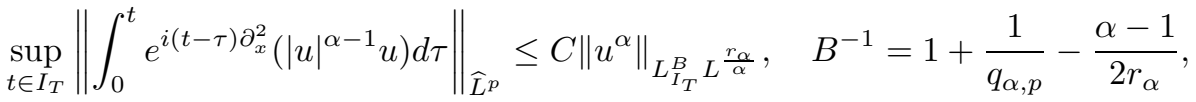

since $\frac{2}{B^{\prime}}+\frac{1}{\left(\frac{r_{\alpha}}{\alpha}\right)^{\prime}}=\frac{1}{p^{\prime}}$. Therefore, the $C\left([0, T]: \widehat{L}^{p}\right)$-norm of the nonlinear part is smaller than $C T^{\beta}\|u\|_{L_{[0, T]}^{q_{\alpha, p} L^{r_{\alpha}}}}^{\alpha}$ by Hölder's inequality. This completes the proof of (i).

The proof of (ii) proceeds similarly. We put $r=\alpha+1$ for any $\alpha$. Obviously, we have $\left\|e^{i t \partial_{x}^{2}} u_{0}\right\|_{\mathcal{V}^{q, \alpha+1}} \leq C\left\|u_{0}\right\|_{\widehat{L}^{p}}$ by Lemma 3 . For the $L_{[0, T]}^{q} L^{r}$-norm of the nonlinear part we get an upper bound $T^{1-\frac{\alpha-1}{2(\alpha+1)}-\frac{\alpha-1}{q}}\|u\|_{L_{I_{T}}^{q} L^{\alpha+1}}^{\alpha}$ in the same way. Finally, the duality of (9) yields

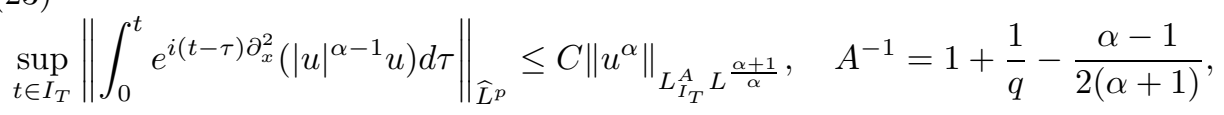

since $\frac{2}{A^{\prime}}+\frac{1}{\left(\frac{\alpha+1}{\alpha}\right)^{\prime}}=\frac{1}{p^{\prime}}$ and the assumption $\frac{1}{2}>\frac{1}{p}=\frac{2}{q}+\frac{1}{\alpha+1}>\max \left(\frac{\alpha-1}{2(\alpha+1)}, \frac{1}{\alpha+1}\right)$ implies $\left(\frac{1}{\alpha+1}, \frac{1}{A^{\prime}}\right) \in \widehat{T}_{3}$. Hence we once again obtain an upper bound

$$
T^{1-\frac{\alpha-1}{2(\alpha+1)}-\frac{\alpha-1}{q}}\|u\|_{L_{[0, T]}^{q}}^{\alpha} L^{\alpha+1}
$$

for (23), which completes the proof of (ii).

\section{Proof of Theorem 2}

In this section, we give the proof of our global result. We only prove the case of the plus sign in $\left(\mathbf{N L S} \mathbf{S}^{\alpha}\right)$ and construct a solution in $t>0$. We mainly prove (ii); the assertion (i) can be proven in a similar manner (see the last part of the section). In what follows we assume that the initial data $u_{0}$ satisfies $u_{0} \in \widehat{L}^{p}(\mathbb{R}) \backslash L^{2}(\mathbb{R})$. Let $p_{0}$ be such that $p<p_{0}<\alpha+1$. We write

$$
\frac{1}{p^{\prime}}=\frac{1}{p_{\theta}^{\prime}}:=\frac{1-\theta}{p_{0}^{\prime}}+\frac{\theta}{2}, \quad 0<\theta<1 \text {. }
$$

4.1. Interpolation of $u$ between $L^{2}$ and $\widehat{L}^{p_{0}}$. Our first step of the proof is to decompose the initial data $u_{0}$ in $L^{2}+\widehat{L}^{p_{0}}$ so that the size of the $\widehat{L}^{p_{0}}$-data can be controlled by any arbitrary small quantity: 
Step 1. There exist a positive constant $C_{0}$ and sequences $\left(\varphi_{N}\right)_{N>1} \subseteq L_{x}^{2}$ and $\left(\psi_{N}\right)_{N>1} \subseteq \widehat{L}_{x}^{p_{0}}$ indexed by $(1, \infty)$ such that for any $N>1$ we have

$$
u_{0}=\varphi_{N}+\psi_{N}
$$

and

$$
C_{0}^{-1} N^{\frac{1-\theta}{\theta}} \leq\left\|\varphi_{N}\right\|_{L^{2}} \leq C_{0} N^{\frac{1-\theta}{\theta}}, \quad\left\|\psi_{N}\right\|_{\widehat{L}^{p_{0}}} \leq C_{0} \frac{1}{N}
$$

Proof of Step 1. By the assumption, $\hat{u}_{0} \in L^{p_{\theta}^{\prime}}$. We apply Lemma 10] with $f=$ $\hat{u}_{0}, q_{0}=p_{0}^{\prime}, q_{1}=2$. Then, there exist $c_{0},\left(v_{0}^{t}\right)_{t>0} \subseteq L^{2}$ and $\left(w_{0}^{t}\right)_{t>0} \subseteq L^{p_{0}^{\prime}}$ such that $\hat{u}_{0}=v_{0}^{t}+w_{0}^{t}$ and

$$
c t^{-\theta} \max \left\{\left\|w_{0}^{t}\right\|_{L^{p_{0}^{\prime}}}, t\left\|v_{0}^{t}\right\|_{L^{2}}\right\} \leq\left\|\hat{u}_{0}\right\|_{L^{p_{\theta}^{\prime}}}
$$

for all $t>0$. Note that $\left\|v_{0}^{t}\right\|_{L^{2}} \rightarrow \infty$ as $t \rightarrow 0$, since $\hat{u}_{0} \notin L^{2}$. Therefore, we can choose $t_{N}>0$ for any $N>1$ such that

$$
\left\|v_{0}^{t_{N}}\right\|_{L^{2}}=N^{\frac{1-\theta}{\theta}}
$$

Let us define

$$
\varphi_{N}^{\prime}:=v_{0}^{t_{N}}, \psi_{N}^{\prime}:=w_{0}^{t_{N}}
$$

Then, we get by (20),

$$
t_{N}^{1-\theta} \leq\left\|\hat{u}_{0}\right\|_{L^{p_{\theta}^{\prime}}} N^{-\frac{1-\theta}{\theta}} .
$$

Thus, by (20) again, we have

$$
\left\|\psi_{N}^{\prime}\right\|_{L^{p_{0}^{\prime}}} \leq t_{N}^{\theta}\left\|\hat{u}_{0}\right\|_{L^{p_{\theta}^{\prime}}} \leq\left\|\hat{u}_{0}\right\|_{L^{p_{\theta}^{\prime}}}^{\frac{1}{1-\theta}} \cdot N^{-1} .
$$

Now, if we put

$$
\widehat{\varphi}_{N}(\xi):=\varphi_{N}^{\prime}(\xi), \widehat{\psi}_{N}(\xi):=\psi_{N}^{\prime}(\xi),
$$

we get the wanted decomposition.

4.2. Local existence to a time $T \sim N^{-\frac{4(\alpha-1)}{5-\alpha} \cdot \frac{1-\theta}{\theta}}$. Let $Q$ be such that

$$
\frac{2}{Q}+\frac{1}{\alpha+1}=\frac{1}{p_{0}}
$$

Moreover, we introduce some constants. We first fix $M>1$ satisfying

$$
M>\max \left\{K_{2},\left(3^{\frac{1}{\alpha-1}} C_{3}^{\frac{1}{\alpha-1}} K_{1}\right)^{\frac{5-\alpha}{4}}, 2^{-1} \cdot 4^{\frac{\alpha}{\sigma_{\alpha}}} C_{0}^{\frac{\alpha-1}{\sigma_{\alpha}}-1} C_{1}^{\frac{\alpha-1}{\sigma_{\alpha}}} C_{3}^{\frac{1}{\sigma_{\alpha}}}\right\},
$$

where $C_{0}, C_{1}, C_{3}, K_{i}(i=1,2)$ are constants in (26), (7), (12), and Proposition 9 respectively and

$$
\sigma_{\alpha}:=\frac{4(\alpha-1)}{5-\alpha}\left(1-\frac{\alpha-1}{2(\alpha+1)}-\frac{\alpha-1}{Q}\right)>0 .
$$

Now for $N>1$ we define $\delta_{N}$ by

$$
\delta_{N}:=\left(M\left(2 C_{0} N^{\frac{1-\theta}{\theta}}\right)\right)^{-\frac{4(\alpha-1)}{5-\alpha}} .
$$

The purpose of Step 2 is to prove the existence of a solution on $\left[0, \delta_{N}\right]$. 
Step 2. For any $N>1$ there exists a unique solution $u$ of $\left(\mathbf{N L S} \mathbf{S}^{\alpha}\right)$ of the form

$$
u=v+w \in L_{I_{\delta_{N}}}^{\frac{4(\alpha+1)}{\alpha-1}} L^{\alpha+1}+L_{I_{\delta_{N}}}^{Q} L^{\alpha+1} .
$$

Moreover, there is $C>0$ such that we have

$$
\left\|w(t)-e^{i t \partial_{x}^{2}} \psi_{0}^{N}\right\|_{L_{x}^{2}} \leq C N^{-1+\left\{-\frac{(\alpha-1)^{2}}{(5-\alpha)(\alpha+1)}+\frac{4(\alpha-1)}{(5-\alpha) Q}\right\} \frac{1-\theta}{\theta}} \quad \text { uniformly in } N
$$

for any $t \in\left[0, \delta_{N}\right]$.

Proof of Step 2. Fix $N>1$. Instead of (26), we construct a solution of $\left(\mathbf{N L S}^{\alpha}\right)$ under the weaker assumption

$$
\left(2 C_{0}\right)^{-1} N^{\frac{1-\theta}{\theta}} \leq\left\|\varphi_{0}^{N}\right\|_{L_{x}^{2}} \leq\left(2 C_{0}\right) N^{\frac{1-\theta}{\theta}}, \quad\left\|\hat{\psi}_{N}\right\|_{L^{p_{0}^{\prime}}} \leq C_{0} \frac{1}{N}
$$

for later use in the next step. The solution $u$ can be expressed as $u=w+v$, where $v, w$ satisfy

$$
\left\{\begin{array}{l}
i v_{t}+v_{x x}+|v|^{\alpha-1} v=0, \quad t \in \mathbb{R} \\
v(0, x)=\varphi_{0}^{N}(x), \quad x \in \mathbb{R}
\end{array}\right.
$$

and

$$
\left\{\begin{array}{l}
i w_{t}+w_{x x}+\tilde{G}(v, w, 0)=0, \quad t \in \mathbb{R}, \\
w(0, x)=\psi_{0}^{N}(x), \quad x \in \mathbb{R} .
\end{array}\right.
$$

It is well-known that $v$ is defined globally (see [10]) and

$$
\|v(t, \cdot)\|_{L_{x}^{2}}=\left\|\varphi_{0}^{N}\right\|_{L_{x}^{2}}
$$

for all $t \geq 0$. Therefore, we will prove that $w$ is well-defined on $\left[0, \delta_{N}\right]$. Since $M>K_{2}$, we have

$$
\|v\|_{L_{I_{\delta_{N}}}^{\frac{4(\alpha+1)}{\alpha-1}}} \leq K_{1}\left\|\varphi_{0}^{N+1}\right\|_{L_{x}^{2}}
$$

by Proposition 9. We use the fixed point theorem in the complete metric space $\mathcal{V}_{N}$ defined by

$$
\mathcal{V}_{N}:=\left\{w \in L_{I_{\delta_{N}}}^{Q} L^{\alpha+1}:\|w\|_{L_{I_{\delta_{N}}}^{Q} L^{\alpha+1}} \leq \frac{3 C_{0} C_{1}}{N}\right\},
$$

where $C_{1}$ is the constant in (7). We prove:

Claim. Define

$$
(T w)(t):=e^{i t \partial_{x}^{2}} \psi_{0}^{N}+i \int_{0}^{t} e^{i(t-\tau) \partial_{x}^{2}} \tilde{G}(v, w, 0) d \tau .
$$

Then, the operator $T: \mathcal{V}_{N} \rightarrow \mathcal{V}_{N}$ is well-defined and is a contraction mapping. 
Proof of Claim. Let $w \in L_{I_{\delta_{N}}}^{Q} L^{\alpha+1}$. Then, by (12) we have

$$
\begin{aligned}
\|T w\|_{L_{I_{\delta}}^{Q} L^{\alpha+1}} \leq & \left\|e^{i t \partial_{x}^{2}} \psi_{0}^{N}\right\|_{L_{I_{\delta_{N}}}^{Q} L^{\alpha+1}}+\left\|\int_{0}^{t} e^{i(t-\tau) \partial_{x}^{2}} \tilde{G}(v, w, 0) d \tau\right\|_{L_{I_{\delta_{N}}}^{Q} L^{\alpha+1}} \leq\left\|e^{i t \partial_{x}^{2}} \psi_{0}^{N}\right\|_{L_{I_{\delta_{N}}}^{Q} L^{\alpha+1}}+C_{3}\left(\delta_{N}\right)^{\frac{5-\alpha}{4}}\|v\|_{L_{I_{\delta_{N}}}^{\alpha-1}{ }^{\frac{4(\alpha+1)}{\alpha-1}} L^{\alpha+1}}\|w\|_{L_{I_{\delta_{N}}}^{Q} L^{\alpha+1}} \\
& +C_{3}\left(\delta_{N}\right)^{1-\frac{\alpha-1}{2(\alpha+1)}-\frac{\alpha-1}{Q}}\|w\|_{L_{I_{\delta_{N}}}^{Q} L^{\alpha+1}}^{\alpha} .
\end{aligned}
$$

For the first term of the right-hand side we have

$$
\left\|e^{i t \partial_{x}^{2}} \psi_{0}^{N}\right\|_{L_{I_{\delta_{N}}}^{Q} L^{\alpha+1}} \leq C_{1}\left\|\widehat{\psi_{0}^{N}}\right\|_{L^{p_{0}^{\prime}}} \leq \frac{C_{0} C_{1}}{N}
$$

using (7) and (26) .

The second term is bounded above by

$$
\begin{aligned}
& C_{3}\left(\left\{M\left(2 C_{0} N^{\frac{1-\theta}{\theta}}\right)\right\}^{-\frac{4(\alpha-1)}{5-\alpha}}\right)^{\frac{5-\alpha}{4}} \times\left(K_{1}\left\|\varphi_{0}^{N}\right\|\right)^{\alpha-1} \times \frac{3 C_{0} C_{1}}{N} \\
\leq & 3 C_{3} K_{1}^{\alpha-1} M^{\frac{4(\alpha-1)}{5-\alpha}} \times \frac{C_{0} C_{1}}{N} \\
\leq & \frac{C_{0} C_{1}}{N}
\end{aligned}
$$

where the last inequality follows from the definition of $M$.

Similarly, we have

The third term $\leq C_{3}\left\{M\left(2 C_{0} N^{\frac{1-\theta}{\theta}}\right)\right\}^{-\frac{4(\alpha-1)}{5-\alpha} \cdot\left(1-\frac{\alpha-1}{2(\alpha+1)}-\frac{\alpha-1}{Q}\right)} \times\left(\frac{3 C_{0} C_{1}}{N}\right)^{\alpha}$

$$
\begin{aligned}
& \leq C_{3} M^{-\sigma_{\alpha}}\left(2 C_{0}\right)^{-\sigma_{\alpha}} \cdot 3^{\alpha} C_{0}^{\alpha-1} C_{1}^{\alpha-1} \times \frac{C_{0} C_{1}}{N} \\
& \leq \frac{C_{0} C_{1}}{N} .
\end{aligned}
$$

Therefore, we obtain

$$
\|T w\|_{L_{I_{\delta_{N}}}^{Q} L^{\alpha+1}} \leq \frac{C_{0} C_{1}}{N}+\frac{C_{0} C_{1}}{N}+\frac{C_{0} C_{1}}{N} \leq \frac{3 C_{0} C_{1}}{N},
$$

which implies $T w \in \mathcal{V}_{N}$. 
In a similar manner, we can prove that $T$ is a contraction mapping. Let $w_{1}, w_{2} \in$ $\mathcal{V}_{N}$. Then, we have

$$
\begin{aligned}
\left\|T w_{1}-T w_{2}\right\|_{L_{I_{\delta}}^{Q} L^{\alpha+1}} \leq & C_{3}\left[\left(\delta_{N}\right)^{\frac{5-\alpha}{4}}\|v\|_{L_{I_{\delta_{N}}}^{\frac{4(\alpha+1)}{\alpha-1}}}^{\alpha+\alpha+1}\right. \\
& +\left(\delta_{N}\right)^{1-\frac{\alpha-1}{2(\alpha+1)}-\frac{\alpha-1}{Q}}\left\|w_{1}\right\|_{L_{I_{\delta_{N}}}^{Q} L^{\alpha+1}}^{\alpha-1} \\
& \left.+\left(\delta_{N}\right)^{1-\frac{\alpha-1}{2(\alpha+1)}-\frac{\alpha-1}{Q}}\left\|w_{2}\right\|_{L_{I_{\delta_{N}}}^{Q}}^{\alpha-1} L^{\alpha+1}\right] \times\left\|w_{1}-w_{2}\right\|_{L_{I_{\delta_{N}}}^{Q} L^{\alpha+1}} \\
\leq & C_{3}\left[K_{1}^{\alpha-1} M^{-\frac{4(\alpha-1)}{5-\alpha}}+2\right. \\
& \left.\times\left(\frac{3 C_{0} C_{1}}{N}\right)^{\alpha-1}\right] \times\left\|w_{1}-w_{2}\right\|_{L_{I_{\delta_{N}}}^{Q}} L^{\alpha+1} \\
\leq & \left(\frac{1}{3}+2 \times \frac{1}{4}\right)\left\|w_{1}-w_{2}\right\|_{L_{I_{\delta_{N}}}^{Q} L^{\alpha+1}}=\frac{5}{6}\left\|w_{1}-w_{2}\right\|_{L_{I_{\delta_{N}}}^{Q} L^{\alpha+1}} .
\end{aligned}
$$

Thus, the claim is proved and we have a solution of $\left(\mathbf{N L S}^{\alpha}\right)$ on $\left[0, \delta_{N}\right]$ by using Duhamel's principle and the fixed point theorem. One can also prove (31) in a similar way, using (16) instead of (12).

\subsection{Global existence.}

Step 3. One can extend $u(t), t \in\left[0, \delta_{N}\right]$ obtained in the previous step to the time $T_{N}$ defined by

$$
T_{N}:=c k_{0} \delta_{N}=c N^{1-\left\{\frac{2\left(2 \alpha^{2}-\alpha-5\right)}{(5-\alpha)(\alpha+1)}+\frac{4(\alpha-1)}{(5-\alpha) Q}\right\} \cdot \frac{1-\theta}{\theta},}
$$

where $c>0$ is a constant independent of $N$. Moreover, if $\alpha, p$ satisfy (6), $u$ can be extended globally by taking $Q$ sufficiently large.

Proof of Step 3. We write

$$
u\left(\delta_{N}, x\right)=\varphi_{1}^{N}(x)+\psi_{1}^{N}(x)
$$

with

$$
\varphi_{1}^{N}(x):=v\left(\delta_{N}, x\right)+\int_{0}^{\delta_{N}} e^{i(t-\tau) \partial_{x}^{2}} \tilde{G}(v, w, 0)(\cdot, \tau) d \tau
$$

and

$$
\psi_{1}^{N}(x):=e^{i \delta_{N} \partial_{x}^{2}} \psi_{0}^{N}
$$

Obviously, we have

$$
\left\|\widehat{\psi_{1}^{N}}\right\|_{L^{p_{0}^{\prime}}}=\left\|e^{i \delta_{N} \xi^{2}} \widehat{\psi_{0}^{N}}(\xi)\right\|_{L_{\xi}^{p_{0}^{\prime}}}=\left\|\widehat{\psi_{0}^{N}}\right\|_{L^{p_{0}^{\prime}}} \leq \frac{C_{0}}{N} .
$$

Therefore, if the estimate

$$
\left(2 C_{0}\right)^{-1} N^{\frac{1-\theta}{\theta}} \leq\left\|\varphi_{1}^{N}\right\|_{L_{x}^{2}} \leq\left(2 C_{0}\right) N^{\frac{1-\theta}{\theta}}
$$


is valid, we get the decomposition of $u\left(\delta_{N}, \cdot\right)$ as in Step 1, and then we obtain the solution of

$$
\left\{\begin{array}{l}
i u_{t}+u_{x x}+|u|^{\alpha-1} u=0, \quad t>\delta_{N}, \\
u\left(\delta_{N}, x\right)=\varphi_{1}^{N}(x)+\psi_{1}^{N}(x), \quad x \in \mathbb{R},
\end{array}\right.
$$

on $\left[\delta_{N}, 2 \delta_{N}\right]$, repeating the calculation of Step 2. In this way, we can construct a solution of $\left(\mathbf{N L S}^{\alpha}\right)$ to the time $2 \delta_{N}, 3 \delta_{N}, \cdots, k_{0} \delta_{N}$ inductively as far as

$$
\left(2 C_{0}\right)^{-1} N^{\frac{1-\theta}{\theta}} \leq\left\|\varphi_{k}^{N}\right\|_{L_{x}^{2}} \leq\left(2 C_{0}\right) N^{\frac{1-\theta}{\theta}}, \quad \forall k \leq k_{0},
$$

where

$$
\varphi_{k}^{N}(x):=v\left(k \delta_{N}, x\right)+\int_{0}^{k \delta_{N}} e^{i(t-\tau) \partial_{x}^{2}} \tilde{G}(v, w, 0)(\cdot, \tau) d \tau
$$

and

$$
\psi_{k}^{N}(x):=e^{i k \delta_{N} \partial_{x}^{2}} \psi_{0}^{N} .
$$

We seek the largest $k_{0}$ for which (43) holds. By (31) and the conservation law (35), if

$$
C_{0} N^{\frac{1-\theta}{\theta}}+C k_{0} N^{-1+\left\{-\frac{(\alpha-1)^{2}}{(5-\alpha)(\alpha+1)}+\frac{4(\alpha-1)}{(5-\alpha) Q}\right\} \frac{1-\theta}{\theta}} \leq 2 C_{0} N^{\frac{1-\theta}{\theta}}
$$

and

$$
C_{0}^{-1} N^{\frac{1-\theta}{\theta}}-C k_{0} N^{-1+\left\{-\frac{(\alpha-1)^{2}}{(5-\alpha)(\alpha+1)}+\frac{4(\alpha-1)}{(5-\alpha) Q}\right\} \frac{1-\theta}{\theta}} \geq\left(2 C_{0}\right)^{-1} N^{\frac{1-\theta}{\theta}},
$$

we obtain (43). Solving (44), (45), we have

$$
k_{0} \leq C N^{1+\left\{\frac{2(\alpha+3)}{(5-\alpha)(\alpha+1)}-\frac{4(\alpha-1)}{(5-\alpha) Q}\right\} \frac{1-\theta}{\theta}} .
$$

So, we can extend the solution of $\left(\mathbf{N L S}^{\alpha}\right)$ to the time

$$
T_{N}:=c k_{0} \delta_{N}=c N^{1-\left\{\frac{2\left(2 \alpha^{2}-\alpha-5\right)}{(5-\alpha)(\alpha+1)}+\frac{4(\alpha-1)}{(5-\alpha) Q}\right\} \cdot \frac{1-\theta}{\theta}} .
$$

Note that $N$ can be taken arbitrarily large, which means that if the exponent of the right-hand side of (46) is strictly positive, $T_{N}$ also can be arbitrarily large. Therefore, if

$$
1-\left\{\frac{2\left(2 \alpha^{2}-\alpha-5\right)}{(5-\alpha)(\alpha+1)}+\frac{4(\alpha-1)}{(5-\alpha) Q}\right\} \cdot \frac{1-\theta}{\theta}>0,
$$

we extend a solution of $\left(\mathbf{N L S}^{\alpha}\right)$ globally. Since $Q$ can be a sufficiently large number (accordingly, $p_{0}$ becomes close to $\alpha+1$ ), if we solve

$$
1-\left\{\frac{2\left(2 \alpha^{2}-\alpha-5\right)}{(5-\alpha)(\alpha+1)}\right\} \cdot \frac{1-\theta}{\theta}>0,
$$

and (24) with $p_{0}=\alpha+1$, we get the assertion of Theorem 2 (ii).

Remark 11. Noting that $L_{[-T, T]}^{Q} L^{\alpha+1} \subseteq L_{[-T, T]}^{\frac{4(\alpha+1)}{\alpha-1}} L^{\alpha+1}$ when $Q$ is large, we can easily prove the uniqueness assertion using Proposition 7.

Sketch of Proof of (i). By interpolating $u_{0}$ between $\left(\frac{1}{r_{\alpha}}, \frac{1}{q_{\alpha, 2}}\right) \in \overline{B C}$ and $\left(\frac{1}{r_{\alpha}}, \frac{1}{q_{\alpha}^{\min }}\right)$, we can prove the assertion $(i)$ in the similar manner: Let $u_{0} \in \widehat{L}^{p}$ with $q_{\alpha}^{\text {min }}<q_{\alpha, p}<$ $q_{\alpha, 2}$. We take $\tilde{q}$ so that $q_{\alpha}^{\min }<\tilde{q}<q_{\alpha, p}$ and write

$$
\frac{1}{p^{\prime}}=\frac{1-\theta}{\tilde{p}^{\prime}}+\frac{\theta}{2}, \quad 0<\theta<1,
$$


where $\tilde{p}$ is defined by $\frac{2}{\tilde{q}}+\frac{1}{r_{\alpha}}=\frac{1}{\tilde{p}}$. We can split the initial data in the same way as the proof of Step 1:

$$
u_{0}=\varphi_{N}+\psi_{N}, \quad \text { with } \quad\left\|\varphi_{N}\right\|_{L^{2}} \sim N^{\frac{1-\theta}{\theta}}, \quad\left\|\psi_{N}\right\|_{\widehat{L}^{\tilde{p}}} \lesssim N^{-1}, \quad N>1 .
$$

Then we can construct a local solution to the Cauchy problem to a time $T \sim$ $N^{-\frac{4(\alpha-1)}{5-\alpha)} \cdot \frac{1-\theta}{\theta}}$ :

Step $2^{\prime}$. For any $N>1$ there exists a unique solution $u$ of $\left(\mathbf{N L S}^{\alpha}\right)$ of the form

$$
u=v+w \in L_{I_{\delta_{N}}}^{q_{\alpha, 2}} L^{r_{\alpha}}+L_{I_{\delta_{N}}}^{\tilde{q}} L^{r_{\alpha}} .
$$

Moreover, there is $C>0$ such that we have

$$
\left\|w(t)-e^{i t \partial_{x}^{2}} \psi_{0}^{N}\right\|_{L_{x}^{2}} \leq\left\{\begin{array}{lll}
C N^{-1+\left(\frac{4}{\tilde{q}}-\frac{\alpha-1}{\alpha+1}\right) \cdot \frac{\alpha-1}{5-\alpha} \cdot \frac{1-\theta}{\theta}} & \text { when } & \alpha \in(1,3), \\
C N^{-1+\left(\frac{4}{\tilde{q}}-\frac{1}{2}\right) \cdot \frac{\alpha-1}{5-\alpha} \cdot \frac{1-\theta}{\theta}} & \text { when } & \alpha \in\left[3, \frac{11}{3}\right], \\
C N^{-1+\left(\frac{4}{\tilde{q}}+\frac{11}{6 \alpha}-1\right) \cdot \frac{\alpha-1}{5-\alpha} \cdot \frac{1-\theta}{\theta}} & \text { when } & \alpha \in\left[\frac{11}{3}, 5\right)
\end{array}\right.
$$

for any $t \in\left[0, \delta_{N}\right]$ (note that we can apply Proposition 8 , since $\tilde{q}>q_{\alpha}^{\min }$ ). Then, proceeding as in the proof of Step 3, we can extend the solution in Step $2^{\prime}$ up to the time $T_{N}$ which is defined by

$$
T_{N}:=c N^{\beta(\alpha, \theta)}:=\left\{\begin{array}{lll}
c N^{1+\left(\frac{2\left(-2 \alpha^{2}+\alpha+5\right)}{\alpha+1}-\frac{4(\alpha-1)}{\tilde{q}}\right) \cdot \frac{1}{5-\alpha} \cdot \frac{1-\theta}{\theta}} & \text { when } & \alpha \in(1,3), \\
c N^{1+\left(\frac{17-9 \alpha}{2}-\frac{4(\alpha-1)}{\tilde{q}}\right) \cdot \frac{1}{5-\alpha} \cdot \frac{1-\theta}{\theta}} & \text { when } & \alpha \in\left[3, \frac{11}{3}\right], \\
c N^{1+\left(\frac{-24 \alpha^{2}+37 \alpha+11}{6 \alpha}-\frac{4(\alpha-1)}{\tilde{q}}\right) \cdot \frac{1}{5-\alpha} \cdot \frac{1-\theta}{\theta}} & \text { when } & \alpha \in\left[\frac{11}{3}, 5\right) .
\end{array}\right.
$$

Finally, letting $\tilde{q} \downarrow q_{\alpha}^{\min }$ and solving $\beta(\alpha, \theta)>0$ with (48), we obtain the assertion (i).

\section{ACKNOWLEDGEMENT}

The authors would like to thank the referee for valuable comments and suggestions which substantially improved our paper (especially, the persistence property of the solutions and results for $p<2$ ).

\section{REFERENCES}

[1] J. Bourgain, Global solutions of nonlinear Schrödinger equations, Amer. Math. Soc. Colloquium Publ., vol 46, 1999. MR1691575 (2000h:35147)

[2] T. Cazenave, L. Vega, and M.-C. Vilela, A note on nonlinear Schrödinger equations in weak $L^{p}$ spaces, Commun. Contemp. Math. 3 (2001) 153-162. MR1820017 (2002g:35192)

[3] C. Fefferman, Inequalities for strongly singular convolution operators, Acta Math., 124 (1970) 9-36. MR0257819 (41:2468)

[4] A. Grünrock, An improved local well-posedness result for the modified KdV equation, Int. Math. Res. Not., 61 (2004) 3287-3308. MR2096258 (2006f:35242)

[5] A. Grünrock, Bi- and trilinear Schrödinger estimates in one space dimension with applications to cubic NLS and DNLS, Int. Math. Res. Not., 41 (2005) 2525-2558. MR2181058 (2007b:35298)

[6] R. Hyakuna, T. Tanaka, and M. Tsutsumi, On the global well-posedness for the nonlinear Schrödinger equations with large initial data of infinite $L^{2}$ norm, Nonlinear Analysis TMA 74 (2011) 1304-1319. MR2746809

[7] T. Kato, An $L^{q, r}$-theory for nonlinear Schrödinger equations, Adv. Stud. Pure Math., vol. 23, Math. Soc. Japan, Tokyo, 1994, 223-238. MR1275405 (95i:35276)

[8] T. Kato, Nonlinear Schrödinger Equations, Lecture Notes in Physics, 345, Springer, 1989, 218-263. MR.1037322 (91d:35202) 
[9] H. Triebel, Interpolation Theory, Function Spaces, Differential Operators, North-Holland, Amsterdam-New York-Oxford, 1978. MR503903 (80i:46032b)

[10] Y. Tsutsumi, $L^{2}$-solutions for nonlinear Schrödinger equations and nonlinear groups, Funkcial Ekvac., 30 (1987) 115-125. MR915266 (89c:35143)

[11] M.C. Vilela, Inhomogeneous Strichartz estimates for the Schrödinger equation, Trans. Amer. Math. Soc., 359 (2007) 2123-2136. MR2276614(2008a:35226)

[12] A. Vargas and L. Vega, Global wellposedness for $1 D$ non-linear Schrödinger equation for data with an infinite $L^{2}$ norm, J. Math. Pures Appl., 80 (2001) 1029-1044. MR.1876762 (2002j:35290)

[13] Yi Zhou, Cauchy problem of nonlinear Schrödinger equation with initial data in Sobolev space $W^{s, p}$ for $p<2$, Trans. Amer. Math. Soc., 362 (2010) 4683-4694. MR2645046 (2011b:35436)

Department of Applied Mathematics, School of Science and Engineering, Waseda UNIVERSITY, TOKYO, JAPAN

Department of Applied Mathematics, School of Science and Engineering, Waseda UNIVERSITY, TOKYO, JAPAN 